\title{
Storage Efficiency of Cold-Crystallizing Long-Term Heat Storage Material
}

\author{
Konsta Turunen \\ Department of Mechanical Engineering \\ Aalto University School of Engineering \\ Espoo, Finland \\ konsta.turunen@aalto.fi
}

\author{
Annukka Santasalo-Aarnio \\ Department of Mechanical Engineering \\ Aalto University School of Engineering \\ Espoo, Finland \\ annukka.santasalo@aalto.fi
}

\author{
Ari Seppälä \\ Department of Mechanical Engineering \\ Aalto University School of Engineering \\ Espoo, Finland \\ ari.seppala@aalto.fi
}

\begin{abstract}
Efficient and compact long-term heat storage material would enable effective utilization of renewable energy sources by balancing the long-term variations in production and consumption. However, current materials still require higher storage capacity, efficiency and reliability for large-scale use. Previously, we established that cold-crystallizing material (CCM), which consists of erythritol in a polymer matrix, can reliably store heat over three months without decreasing its storage efficiency. Heat is stored by cooling the melted CCM to deeply supercooled state (storage temperature at 0-10 ${ }^{\circ} \mathrm{C}$ ) and released by heating the material to cold-crystallization temperature, which initiates crystallization (i.e. coldcrystallization). However, if the storage temperature of CCM was increased, stored melting heat would dissipate due to slow crystallization. This paper analyses cold-crystallization rate of CCM, in order to model and predict the storage efficiency at different storage temperatures. This was carried out by measuring the progress of cold-crystallization by differential scanning calorimetry (DSC) under multiple isothermal conditions. The crystallization data was first analysed by applying Avrami approach, to identify the crystallization rate constant. Then, the Arrhenius and the Williams-Landel-Ferry (WLF) models estimated the temperature dependence of the rate constant. DSC measurements yielded a storage efficiency of around 0.74 in the tested temperature range. Time evolution of this storage efficiency predicted with the WLF model corresponds to the experimental data indicating that valid predictions of CCM's storage efficiency can be obtained, when storage temperature and time are known.
\end{abstract}

Keywords - Long-term thermal energy storage, Phase change material, Supercooling, Cold-crystallization, Erythritol

\section{INTRODUCTION}

Large seasonal and diurnal variations in renewable energy production, such as solar irradiation in cold climate areas, impedes the integration of renewable energy into the current energy systems. Therefore, variety of energy storage technologies are developed, but compact and efficient longterm storage is yet to exist. If this type of long-term storage emerged, it would improve the system efficiency and viability of the renewable energy sources. Long-term thermal energy storage (long-term TES) provides attractive means for achieving this goal by balancing the characteristic seasonal variations of the energy sources.

Thermal energy storages (TES) can be categorized in three main classes: sensible heat, phase change and thermochemical storages. Sensible heat storages exploit the heat capacity of a storage medium by increasing its temperature. Current longterm storages are typically large underground sensible heat storages, as the technology is commercially available [1]. Storages based on phase change materials (PCMs) utilize the large amount of heat involved in phase transitions, typically melting and crystallization of the PCM. Therefore, PCMs can reduce the volume of the storage compared to sensible heat storages. However, because of continuous heat loss, sensible heat and conventional PCM storages require large volumes and insulation in order to be feasible in long-term TES applications. This limits the applicability of the current underground TES [1]. Thirdly, thermochemical storages apply chemical reaction to store and release heat. These storages have great potential in long-term TES, as losses during the storage can be minimized by separating the reactants. However, currently thermochemical storages are not yet feasible. Further research is required to overcome impediments related to material properties, such as instable cycling, inefficient charging-discharging process and high cost [2].

When melted PCM is cooled below its melting temperature, it crystallizes or remains in a liquid state and supercools. Because supercooled liquid is in a metastable state, material is prone to crystallize [3]. Nonetheless, if the liquid remains stable against crystallization, the melting heat can be stored in the supercooled state, in principle, as long as needed.

Typically, PCM applications try to prevent supercooling [4]. Nonetheless, supercooling TES is successfully applied in small-scale heating pads utilizing sodium acetate trihydrate (SAT) [5]. Large-scale applications using supercooling SAT has been studied with $200 \mathrm{~kg}$ units [6], [7]. However, reliable operation of the units has not yet been achieved, as spontaneous crystallization occurred in the supercooled state, discharging the storage prematurely. In addition to SAT, a few studies have explored supercooling TES properties of other materials, such as polyols [8], [9], [10], [11] and other salt hydrates [12], [13], [14], [15]. These materials experience the same barriers as SAT in relation to metastability or they lack efficient method of crystallization initiation. Nevertheless, increased stability against crystallization can be achieved by cooling the material below the glass transition temperature $\left(T_{g}\right)$. This has been observed with metal complexes designed for TES [16]. Heat release is initiated by re-heating the material, that is cold-crystallization.

In our previous work [17], cold-crystallizing material (CCM) demonstrated reliable long-term storing of heat in bulk level. Operational principle of CCM is based on stably supercooling the material close to the glass transition 
temperature $\left(T_{g}\right)$. As the temperature of a supercooled liquid approaches $T_{g}$, molecular movement slows down and the time for structural relaxation prolongs abruptly. At this state, crystallization takes place so slow that the melting heat can be stored for months without reduction [17]. If cooling continued below $T_{g}$, supercooled material would transform to glassy state, thus achieving increased stability against crystallization. CCM takes advantage of the strong ion-dipole interactions between erythritol (active PCM) and a polymer matrix enabling stable supercooling even at low cooling rates $(0.05$ $\mathrm{K} / \mathrm{min}$ ) [8]. Re-heating the material to the cold-crystallization temperature initiates crystallization, releasing the stored heat. As the heat is released, the temperature of CCM approaches the melting temperature, unless CCM's temperature is controlled by transferring heat away from the material. Further description on the composition and the operational principle of CCM can be found in the previous studies [8], [17].

Storage efficiency is an essential indicator of the TES performance. Therefore, estimating and predicting the efficiency of a TES material plays a crucial role in determining the feasibility of the storage system. Because of the unique operational principle of CCM, a method for modelling and predicting the storage efficiency of coldcrystallizing TES materials is needed.

Therefore, this paper examines cold-crystallization kinetics of CCM, in order to model and predict the temperature dependence of the storage efficiency in the supercooled state. For this, differential scanning calorimetry (DSC) measured the overall crystallization rate of CCM at isothermal temperatures between $25{ }^{\circ} \mathrm{C}$ and $50{ }^{\circ} \mathrm{C}$. The Avrami model evaluated the DSC data, to determine the crystallization rate constant $\left(k_{c}\right)$. Then, the temperature dependence of $k_{c}$ was estimated using Arrhenius and Williams-Landel-Ferry (WLF) type behaviour. The results indicate that Avrami model corresponds the crystallization rate of CCM in a repeatable manner. Furthermore, WLF equation appears to yield valid storage efficiency predictions, when measurement accuracy is ensured.

\section{MATERIALS AND METHODS}

\section{A. Material preparation}

CCM consist of erythritol in a cross-linked sodium polyacrylate (PAANa) matrix. Preparation of CCM follows the method described by Puupponen and Seppälä [8]. In summary, PAANa was prepared by polymerizing completely neutralized acrylic acid (assay $\geq 99 \%$, supplied by Merck) in aqueous erythritol (technical grade, supplied by Suomen Luontaistukku Ltd., Finland). Sodium hydroxide (assay $\geq 99 \%$, supplied by VWR Chemicals) was used for neutralization. Potassium persulfate (assay $\geq 98 \%$, supplied by VWR Chemicals) initiated polymerization and ethylene glycol dimethacrylate (assay $\geq 97.5 \%$, supplied by Merck) was used as a cross-linking agent.

The composition of CCM affects its supercooling and cold-crystallization behavior. This study analyses one composition of CCM, which contains by mass fraction $80 \mathrm{wt}-$ $\%$ erythritol, 17.9 wt- $\%$ PAANa, 2 wt- $\%$ ethylene glycol dimethacrylate and $0.1 \mathrm{wt}-\%$ potassium persulfate.

\section{B. DSC measurements}

Differential scanning calorimetry (Netzsch DSC204F1 Phoenix DSC) measured isothermal crystallization rate of CCM. The temperature program used in the measurements consisted of four steps. First, dynamic heating step heated $\mathrm{CCM}$ to $135^{\circ} \mathrm{C}$ with scan rate of $5 \mathrm{~K} / \mathrm{min}$, in order to melt the sample and erase thermal history. Then, the sample was brought to the glassy state, by cooling it to $-60^{\circ} \mathrm{C}$ with the rate of $5 \mathrm{~K} / \mathrm{min}$. The third step heated the sample quickly (20 $\mathrm{K} / \mathrm{min}$ ) to the isothermal crystallization temperature. Lastly, isothermal step maintained the sample in a constant temperature until the cold-crystallization was completed. This program was repeated at least two times for each isothermal crystallization temperature, which ranged from $25{ }^{\circ} \mathrm{C}$ to 50 ${ }^{\circ} \mathrm{C}$. Two samples was used in the DSC measurements. They are referred as CCM-1 and CCM-2, as they were prepared from separate batches. The sample masses were $25.06 \mathrm{mg}$ and $25.16 \mathrm{mg}$ for CCM-1 and CCM-2.

\section{RESULTS AND DISCUSSION}

\section{A. Isothermal crystallization rate}

Isothermal crystallization rate of CCM was measured with DSC. Because DSC records the heat flow, the combined effect of nucleation and crystal growth is analysed. For this type of kinetics, Johnson-Mehl-Avrami-Kolmogorov theory is typically used [18]. Applying extended volume approach to the theory, widely used Avrami equation can be derived as formulated in Eq. (1) and Eq. (2) [19], [20]:

$$
\begin{gathered}
\alpha=1-\exp \left(-K t^{n}\right) \\
\log [-\ln (1-\alpha(t))]=n \log (t)+\log (K) \\
\alpha(t)=\frac{X_{c}(t) \rho_{1}}{\rho_{\mathrm{c}}-X_{c}(t)\left(\rho_{\mathrm{c}}-\rho_{1}\right)}
\end{gathered}
$$

where $\alpha(t)$ is volume-based relative crystallinity $(-), t$ is time from the beginning of the crystallization (s), $K$ a parameter dependent on the nucleation rate and the growth rate and $n$ a parameter describing the type of nucleation and the dimensionality of the crystallization process. Because DSC yields mass-based relative crystallinity, $X_{c}(t)(-)$, it is transformed to $\alpha(t)$ applying Eq. (3), where $\rho$ is density $\left(\mathrm{kg} / \mathrm{m}^{3}\right)$ and subscript $l$ refers to the liquid and $c$ to the crystal phase. Density of $1460 \mathrm{~kg} / \mathrm{m}^{3}$ and $1430 \mathrm{~kg} / \mathrm{m}^{3}$ measured at 25 ${ }^{\circ} \mathrm{C}$ was used for the solid and liquid phase [17].

Eq. (2) can be used to graphically solve the parameters $K$ and $n$. In this case, relative crystallinity data can be presented in a form, where $\log [-\ln (1-\alpha(t))]$ is a function of $\log (t)$. If the relative crystallinity data behaves according to the Avrami equation, a straight line would be displayed. Therefore, the graph conveniently enables determining constants $\ln K$ and $n$ from the interception and the slope of the plotted data, as shown in Fig. 1. However, because of the double-logarithm formulation of the graph, the plotted data is not sensitive for deviations from the straight line. Consequently, R-squared value of the fitted line is recommended to be over 0.9990 , to represent very good fit of the data [21].

Eq. (2) rarely describes the entire crystallization process from the first nuclei until the sample has completely crystallized. Typically, the beginning and the end of the 
Table 1. Thermophysical properties and Avrami parameters in the tested temperature range. All values listed are average values.

\begin{tabular}{|c|c|c|c|c|c|c|c|c|c|}
\hline & \multicolumn{5}{|c|}{ Thermophysical properties } & \multicolumn{4}{|c|}{ Avrami parameters } \\
\hline & $\begin{array}{c}\boldsymbol{T}_{\boldsymbol{m}} \\
{\left[{ }^{\circ} \boldsymbol{C}\right]}\end{array}$ & $\begin{array}{l}\Delta \boldsymbol{H}_{\boldsymbol{m}} \\
{[J / g]}\end{array}$ & $\begin{array}{c}\Delta \boldsymbol{H}_{\boldsymbol{c}} \\
{[\mathrm{J} / \mathrm{g}]^{*}}\end{array}$ & $\begin{array}{c}\boldsymbol{T}_{\boldsymbol{g}} \\
{\left[{ }^{\circ} \boldsymbol{C}\right]}\end{array}$ & $\begin{array}{c}\Delta \boldsymbol{H}_{c} / \Delta \boldsymbol{H}_{\boldsymbol{m}} \\
{[-]}\end{array}$ & $\begin{array}{c}\boldsymbol{T}_{\boldsymbol{c}} \\
{\left[{ }^{\circ} \mathrm{C}\right]}\end{array}$ & $\begin{array}{c}k_{c} \\
{\left[10^{-4} / s\right]}\end{array}$ & $n$ & $R^{2}$ \\
\hline \multirow[t]{5}{*}{ CCM-1 } & 105.7 & 183.4 & 134.5 & -17.7 & 0.73 & 45 & 7.65 & 2.19 & 0.9981 \\
\hline & & & & & & 40 & 4.33 & 2.20 & 0.9986 \\
\hline & & & & & & 35 & 2.42 & 2.29 & 0.9989 \\
\hline & & & & & & 30 & 1.22 & 2.19 & 0.9982 \\
\hline & & & & & & 25 & 0.58 & 1.99 & 0.9980 \\
\hline \multirow[t]{5}{*}{$C C M-2$} & 103.8 & 183.0 & 138.4 & -19.1 & 0.76 & 45 & 7.54 & 2.44 & 0.9999 \\
\hline & & & & & & 40 & 3.96 & 2.52 & 0.9996 \\
\hline & & & & & & 35 & 2.18 & 2.37 & 0.9997 \\
\hline & & & & & & 30 & 1.15 & 2.44 & 0.9972 \\
\hline & & & & & & 27 & 0.71 & 2.59 & 0.9980 \\
\hline
\end{tabular}

crystallization data are excluded from the graphical determination of Avrami parameters, because of the device's measurement accuracy. Moreover, if crystallization behaviour changes during the progress of the crystallization, the data will deviate from the straight line. In this case, only a part of the crystallization process ought to be evaluated at once. [21].

Fig. 1 depicts the Avrami equation fitted to the CCM-1 measurement data using the relative crystallinity range of 0.05 to 0.8 . The data shows linear behaviour within the entire range as predicted by the Avrami equation. This was observed for both samples indicating that the crystallization process does not significantly change when crystallization progresses. Therefore, the wide range from 0.05 to 0.8 was applied in the graphical analysis.

Table 1 lists the thermophysical properties and estimated parameters of the Avrami fits for CCM-1 and CCM-2. The thermophysical properties are in line with the previous study [17] showing that around $74 \%$ of the melting heat is released in cold-crystallization at $45{ }^{\circ} \mathrm{C}$. This released crystallization heat corresponds to the charged melting heat, because the crystallization heat in the supercooled state depends on the temperature at which it takes place and the difference in the specific heat capacity of the liquid and solid state of the

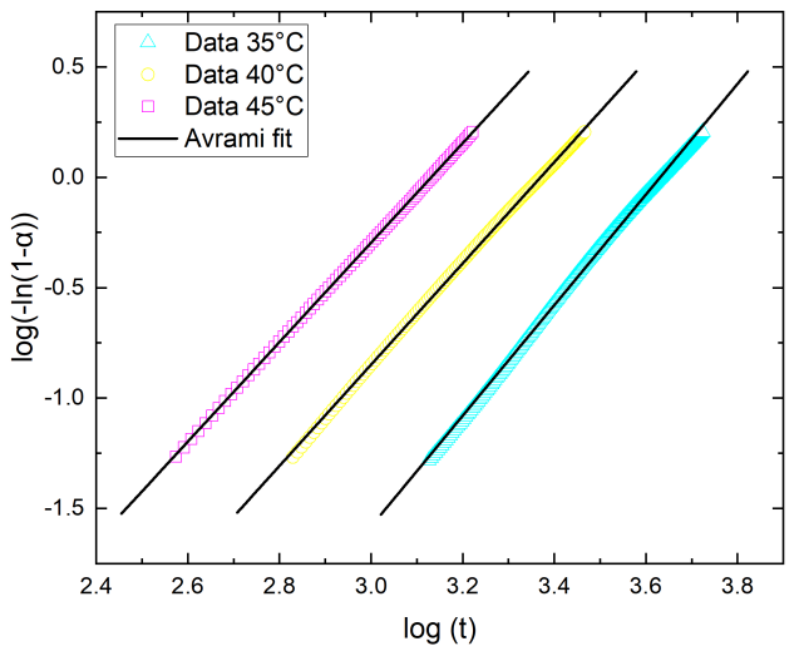

Fig. 1 Avrami plot for CCM-1 measurements at 35, 40 and $45 \mathrm{C}$. The range of crystallization data (points) is 0.05 to 0.8 . The black line shows the fitted avrami equation for the measurement data. material. If the crystallization of CCM would have occurred at the melting temperature, the crystallization heat would be the same as the melting heat.

Both samples show high $R^{2}$ values around 0.9990, which is considered to correspond very good fit [21]. The exponent $n$ experiences some deviation between the measurements. Nonetheless, it does not appear to decrease or increase as a function of temperature indicating that the crystallization process does not significantly change within the tested temperature range from $25^{\circ} \mathrm{C}$ to $45^{\circ} \mathrm{C}$. For CCM-1, the total average value of $n$ equals 2.17 with the mean absolute error of 0.13, while CCM-2 yields 2.47 and 0.15 for the same measures. The difference in the values of $n$ between the samples most likely stem from the preparation of the samples, as they were prepared in separate batches. The deviation between the batches is also visible in the thermophysical values of CCM-1 and CCM-2 listed in Table 1, as the glass transition temperature of CCM-2 is $1.4{ }^{\circ} \mathrm{C}$ lower than CCM1 's. This and the preparation in separate batches most likely causes the difference in the crystallization rate constant $\left(k_{c}\right)$ of CCM-1 and CCM-2. Nonetheless, $k_{c}$ demonstrates expected decreasing trend as a function of temperature, because molecular movement slows down.

Looking at the samples separately, Avrami equation corresponds the crystallization behaviour measured using DSC with small deviation in the values of $n$. As the exponent $n$ describes the dimensionality and the nucleation type of the crystallization process, there are theoretical considerations on the absolute value of $n$. On the other hand, it has been stated that Avrami equation over simplifies the crystallization process, which is shown in varying values of $n$ [22]. Nevertheless, as listed in Table 1, the values for CCM-1 and CCM-2 appear close to the value of 2.5 , which would describe theoretical case of diffusion controlled three-dimensional spherical crystal growth with a constant nucleation rate [23]. Some other cases are listed in the following paper [23]. In reality, crystal growth might not be fully diffusion controlled or perfectly spherical, and nucleation might not occur randomly or steadily in time, resulting in deviations from the theoretical value. In CCM's case, theoretical value of 2.5 appears reasonable, because the DSC samples provide an environment for three-dimensional growth and possibility for constant nucleation. Because of this and the fact that the deviations in $n$ are small, the average value of $n$ was assumed 
Table 2. Arrhenius and WLF fitting parameters for the tested CCM samples.

\begin{tabular}{|c|c|c|c|c|c|c|c|}
\hline & \multicolumn{3}{|c|}{ Arrhenius parameters } & \multicolumn{5}{c|}{ WLF parameters } \\
\cline { 2 - 9 } & $\boldsymbol{E}_{\boldsymbol{a}}[\mathbf{k J} / \mathbf{m o l}]$ & $\boldsymbol{l n}\left(\boldsymbol{k}_{\mathbf{0}}\right)$ & $\boldsymbol{R}^{\mathbf{2}}$ & $\boldsymbol{C}_{1}^{\boldsymbol{g}}[-]$ & $\boldsymbol{C}_{2}^{\boldsymbol{g}}\left[^{\circ} \boldsymbol{C}\right]$ & $\boldsymbol{k}_{\boldsymbol{c}}^{\boldsymbol{g}}[\mathbf{1} / \mathbf{s}]$ & $\boldsymbol{R}^{\mathbf{2}}$ \\
\hline $\mathrm{CCM}-1$ & 101 & 31.2 & 0.9983 & 14.4 & 20.3 & $1.08 * 10^{-14}$ & 0.9996 \\
\hline $\mathrm{CCM}-2$ & 104 & 32.3 & 0.9982 & 12.3 & 81.7 & $2.72 * 10^{-9}$ & 0.9982 \\
\hline
\end{tabular}

as a constant for each sample in the further investigation of the crystallization process. This enables predicting the progress of the crystallization below the tested temperature range, hence predicting the storage efficiency of $\mathrm{CCM}$, which will be discussed in Section III B and Section III C.

\section{B. Temperature dependence of cold-crystallization}

This section analyses the temperature dependence of the crystallization rate constant, $k_{c}$, by applying the Arrhenius and the Williams-Landel-Ferry (WLF) equation. For extrapolating the values of $k_{c}$, the Avrami exponent, $n$, was assumed to remain constant below the temperature range investigated with DSC. Analysis of the exponent, $n$, is discussed in Section III A.

Temperature dependence of a reaction rate constant can be described with Arrhenius equation. The reaction rate constant can be taken as a relevant rate constant, in this case, crystallization rate constant, $k_{c}$ [24]. Therefore, the Arrhenius equation formulates as follows:

$$
k_{c}=k_{0} \exp \left(\frac{-E_{a}}{R T}\right)
$$

where $k_{c}$ is crystallization rate constant $(1 / \mathrm{s}), R$ the universal gas constant $(\mathrm{J} / \mathrm{K} \cdot \mathrm{mol}), T$ temperature $(\mathrm{K})$, and $k_{0}$ $(1 / \mathrm{s})$ and $E_{a}$ fitting parameters. In this case, $E_{a}$ refers to the activation energy for cold-crystallization $(\mathrm{J} / \mathrm{mol})$. The crystallization rate constant is an alternate expression of the constant $K$ in the Avrami equation: $k_{c}=K^{1 / n}$. Eq. (4) states that if $\ln \left(k_{c}\right)$ was plotted against $1 / T$, straight line will form with the slope of $-E_{a} / R$ and the interception at $\ln \left(k_{0}\right)$. Therefore, the fitting parameters can be defined, and activation energy of cold-crystallization can be established.

Viscosities and relaxation times of several glass-forming liquids, deviate from Arrhenius relationship at the temperatures close to glass transition [25]. Therefore, theories and empirical relations describing material's behaviour close to $T_{g}$ has been formulated. Here, we employ WilliamsLandel-Ferry (WLF) equation that is used to explain the relaxation times and the viscosities of supercooled glassforming liquids in the polymer [26] and food sciences [27].

The WLF equation [28] uses the crystallization rate constant at a reference temperature, $T_{s}$, to describe the temperature dependence of the rate constant, $k_{c}$, as a function of temperature and WLF constants $C_{1}$ and $C_{2}$ :

$$
\begin{gathered}
\log \frac{k_{s}}{k_{c}}=\frac{-C_{I}\left(T-T_{s}\right)}{C_{2}+\left(T-T_{s}\right)} \\
\frac{1}{\log \frac{k_{s}}{k_{c}}}=\frac{1}{-C_{l}}-\frac{C_{2}}{C_{l}\left(T-T_{S}\right)} \\
C_{I}^{g}=\frac{C_{l} C_{2}}{C_{2}-\left(T_{s}-T_{g}\right)} \\
C_{2}^{g}=C_{2}-\left(T_{s}-T_{g}\right)
\end{gathered}
$$

Constants $C_{1}$ and $C_{2}$ can be solved graphically using the linearized form of the WLF equation that is Eq. (6). If $1 / \log \frac{k_{s}}{k_{c}}$ is plotted against $1 /\left(T-T_{S}\right)$, straight line forms with the slope of $-C_{2} / C_{1}$ and the interception at $1 /-C_{1}$. Setting the reference temperature at $35^{\circ} \mathrm{C}$ then enables fitting Eq. (6) to the rest of the data using the least squared method. The WLF constants are typically presented using $T_{g}$ as the reference temperature, in order to have comparable results. Therefore, Eq. (7) and Eq. (8) transform the constants to the values that correspond reference temperature at $T_{g}\left(C_{1}^{g}\right.$ and $\left.C_{2}^{g}\right)$.

Table 2 lists the fitting parameters for the Arrhenius and the WLF equations. Arrhenius equation yields similar results for CCM-1 and CCM-2. However, WLF parameters differ considerably as evidenced by the $C_{2}^{g}$ values of $20.3{ }^{\circ} \mathrm{C}$ and $81.7^{\circ} \mathrm{C}$ for CCM-1 and CCM-2. In the WLF equation, $T_{g}-C_{2}^{g}$

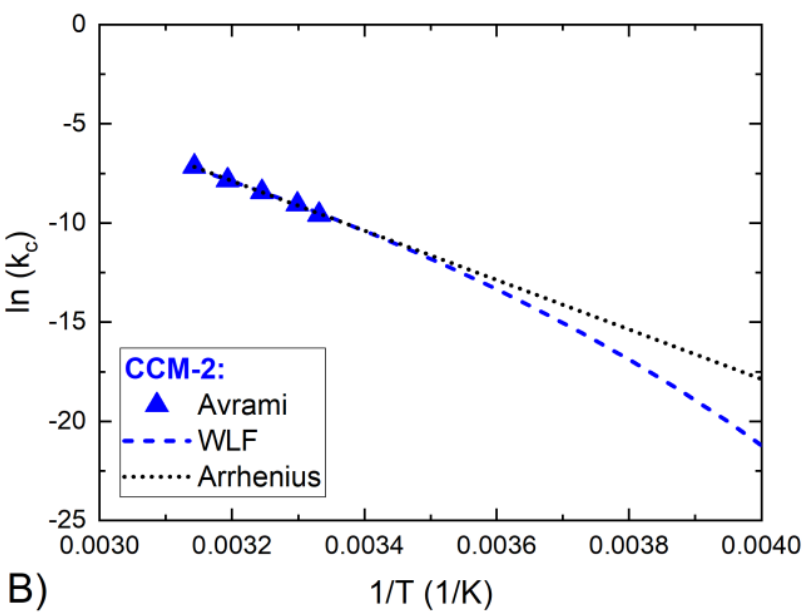

Fig. 2 The temperature dependence of the crystallization rate constant calculated by the Arrhenius and the WLF equations (lines) for CCM-1 (A) and CCM-2 (B). Solid dots represent the crystallization rate constant calculated with Avrami equation based on DSC data. 

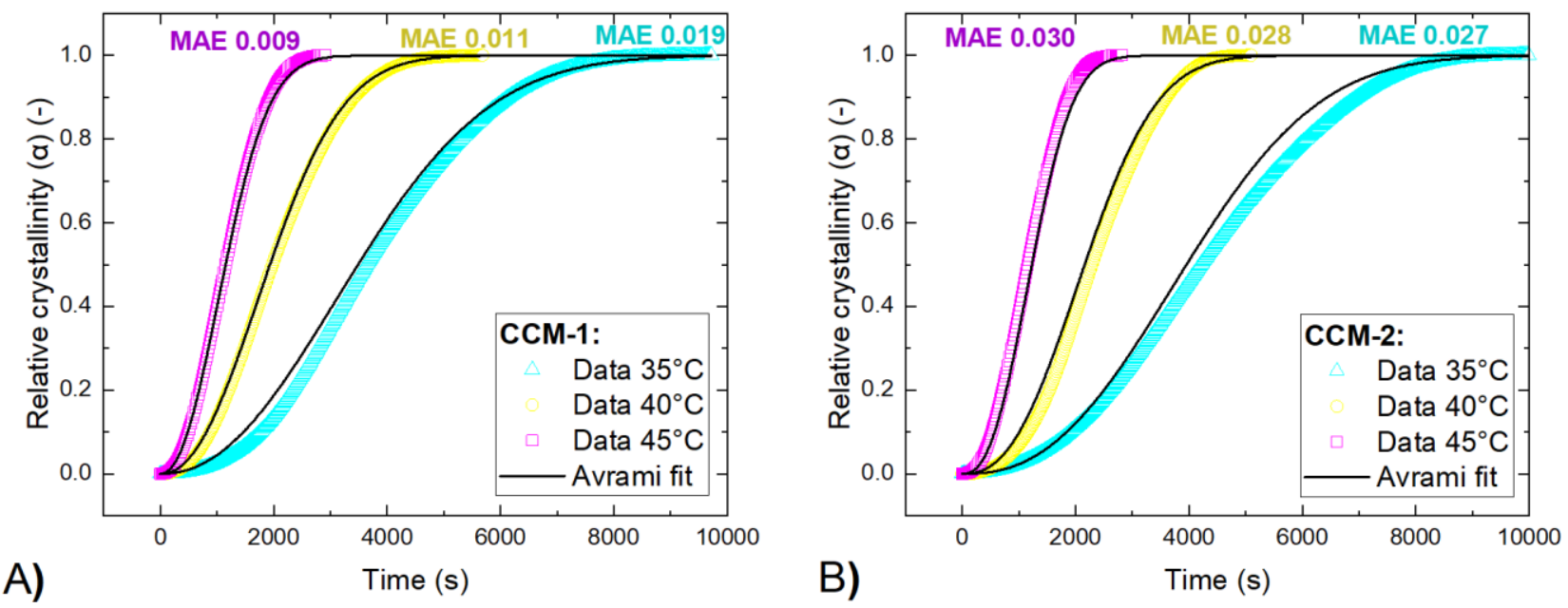

Fig. 3 The time evolution of relative crystallinity of CCM-1 (A) and CCM-2 (B). Coloured points indicate the data measured with DSC. The line represents the modelled values using Avrami equation, for which the WLF equation determined the crystallization rate constant. The mean average error (MAE) is displayed with coloured text.

denotes the temperature at which the crystallization rate constant tends to zero. Therefore, the values of $k_{c}^{g}$ also deviate between the samples, when the temperature decreases towards glass transition temperature. This is illustrated in Fig. 2, where the fitted Arrhenius and WLF equations are plotted with the crystallization rate constants obtained from the Avrami analysis. Because the values of $k_{c}$ do not show strong nonArrhenius behaviour in the tested temperature range, small variations in the $k_{c}$ values may lead to significantly different WLF parameters. The $R^{2}$ values of the WLF fits of CCM-1 and CCM-2 appear reasonable. However, CCM-2 demonstrates lower value indicating decreased accuracy of the fit. In addition, we calculated the mean absolute percentage error (MAPE) for the $k_{c}$ values calculated with the WLF parameters. For CCM-1 and CCM-2, MAPE results in 0.043 and 0.093 , indicating that the WLF model for CCM-2 is more prone to errors.

The accuracy of the WLF model was further examined by comparing the crystallization rate measured by the DSC to the crystallization rate calculated with the WLF fitting parameters while using constant $n$ value in the Avrami equation. The mean average error (MAE) of the WLF model for CCM-1 and CCM-2 equals 0.018 and 0.03 indicating that the models correspond to the measured data. However, as expected by the deviations in $k_{c}, \mathrm{CCM}-2$ shows larger MAE than CCM-1. An example of the modelled time evolution of the crystallization and the DSC data is shown in Fig. 3. The impact of the MAE is exemplified in Fig. 3A, in which the model shows larger deviation from the DSC data at $35{ }^{\circ} \mathrm{C}$ than at other temperatures, resulting in an increased MAE value.

Initially, WLF parameters $C_{1}^{g}$ and $C_{2}^{g}$, which are defined in the reference temperature at $T_{g}$, were proposed to have universal values 17.44 and $51.6{ }^{\circ} \mathrm{C}$ [28]. However, as more materials were investigated, more variations in the values was observed [26]. Nevertheless, the WLF equation has shown correspondence to the measurement data of several systems in the temperature range from $T_{g}$ to $T_{g}+100{ }^{\circ} \mathrm{C}$ [29]. The tested temperature range of $\mathrm{CCM}$ appears to be appropriate, because it is below $T_{g}+65^{\circ} \mathrm{C}$. In terms of the WLF parameters, CCM samples do not match the universal values, as $C_{2}^{g}$ values deviate from $51.6{ }^{\circ} \mathrm{C}$. The values of $C_{l}^{g}$ are closer to the universal value, but notably smaller. As CCM samples have evident difference in the WLF parameters, further measurements ought to be conducted to determine the parameters with higher accuracy.

\section{Storage efficiency}

Storage efficiency is an essential indicator of the storage's performance. In this paper, the storage efficiency of CCM is defined as the ratio of the crystallization heat and melting heat, $\varepsilon=\Delta H_{c} / \Delta H_{m}$. It describes the relation between the discharged heat and the charged heat. In reality, the operational storage efficiency would differ, because the sensible heat during heating and cooling ought to be considered.

The available crystallization heat of CCM, $\Delta H_{c}$, can be estimated using the Avrami equation (Eq. 1), which describes the time evolution of relative crystallinity, as shown in Fig. 3. Therefore, it determines the fraction of the available crystallization heat for discharge (from one to zero). In order to utilize the Avrami equation, the crystallization temperature is set as the storage temperature of CCM. This determines the crystallization rate constant $\left(k_{c}\right)$, which is calculated with the WLF equation or the Arrhenius equation (see. Section III B). Lastly, the exponent $n$ for the Avrami equation was estimated in Section III A. By multiplying the relative crystallinity from the Avrami equation with the initial storage efficiency at zero seconds, the time dependent storage efficiency curve can be formed. The initial storage efficiency was set to 0.74 , because it was reported in our earlier study for the same CCM composition [17] that is well in line with the thermophysical properties of CCM-1 and CCM-2 in Table 1.

In order to evaluate the storage efficiency predictions, they are compared to the previously made long-term storage measurements with $160 \mathrm{~g}$ samples of CCM [17] that have the same composition as in this study. The Arrhenius equation significantly overestimates the crystallization rate, which was expected, because similar CCM compositions have displayed non-Arrhenius behaviour before [8]. Therefore, the WLF model is discussed in detail. 

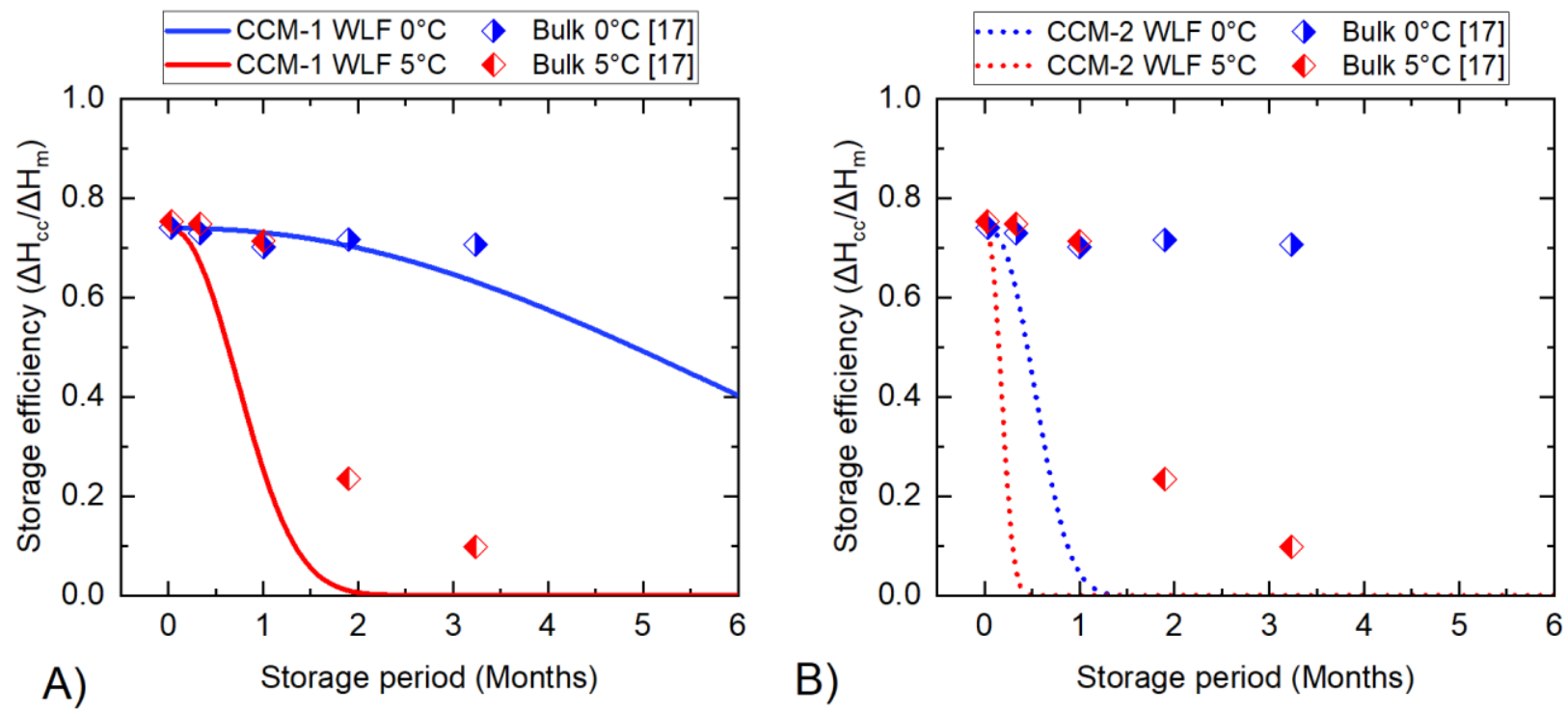

Fig. 4 The storage efficiency of CCM-1 (A) and CCM-2 (B) predicted by the WLF equation (solid and dotted line). Data points are measured values of $160 \mathrm{~g} \mathrm{CCM}$ samples with the same composition as the CCM investigated in this paper [17].

Fig. 4 depicts the experimental data of $160 \mathrm{~g}$ CCM samples and the storage efficiency predictions of CCM samples with the WLF equation. As shown in Fig. 4A, the storage efficiency of CCM-1 decreases earlier than the bulk sample data. Nevertheless, the curvature of the predicted efficiency appears to behave similarly as the data points. In the case of $5^{\circ} \mathrm{C}$ storage temperature, the predicted efficiency shows correspondence to the Bulk $5^{\circ} \mathrm{C}$ data set, if the beginning of the prediction is moved to around three weeks forward in time. This delay can be at least partially explained by the absence of induction period in the predicted values. The method used in this paper analyses the crystallization from the point when it becomes detectable by DSC. On the other hand, the storage period of the bulk samples includes the induction time, which is the time needed for the first nuclei to form. Therefore, the predicted values ought to be moved a duration of the induction time forward to be accurately comparable to the bulk sample data. Considering this, the comparison of the WLF predictions to the bulk data indicates predictable storage behaviour in majority of the relative crystallinity range. Induction time measurements were descoped from this study.

As can be seen in Fig. 4B, the predictions overestimate the crystallization rate of CCM-2 compared to the bulk samples. On the other hand, CCM-1 shows better correspondence to the bulk measurements (Fig. 4A). Because such difference occurs in the predictions, the WLF model appears to be sensitive to the deviations in the DSC measurements and in the Avrami analysis. The sensitive behaviour is most likely caused by the Arrhenius type behaviour of the $k_{c}$ values in the tested temperature range, as illustrated in Fig. 2. This indicates that the $k_{c}$ distinctively diverges from the Arrhenius behaviour at lower temperatures that were tested $\left(45-25{ }^{\circ} \mathrm{C}\right)$. Because CCM-2 has larger deviations in the $k_{c}$ values than CCM-1, as indicated by MAPE in Section III B, the WLF model of CCM2 is more prone to error. This manifests as different values in the WLF constants, especially in $C_{2}^{g}$ values (Table 2). Therefore, CCM-1 yields more reliable results than CCM-2. In order to improve the accuracy of the predictions, crystallization rate ought to be measured at lower temperatures. However, at lower temperatures the detection of crystallization rate becomes laborious, because duration of the crystallization increases abruptly to the timescale of days. Because of this, we ensured the accuracy of the DSC by limiting the continuous crystallization rate measurements to the temperature range from $25^{\circ} \mathrm{C}$ to $45^{\circ} \mathrm{C}$.

The crystallization rate becomes increasingly sensitive to the temperature, when it approaches the glass transition temperature $\left(T_{g}\right)$, as shown by the behaviour of the WLF model for CCM-1 and the bulk sample data in Fig. 4. Therefore, small changes in the $T_{g}$ or in the crystallization temperature can lead to an evident shift in the crystallization rate. This causes some error in the comparison of the bulk data and the predicted values of the WLF model, because the $T_{g}$ values of the samples deviate from one another. The bulk samples in Fig. 4 have $T_{g}$ values of $-16.0^{\circ} \mathrm{C}$ and $-15.2{ }^{\circ} \mathrm{C}$ for Bulk $5^{\circ} \mathrm{C}$ and Bulk $0{ }^{\circ} \mathrm{C}$. In comparison to CCM-1, this leads to a difference of $1.7{ }^{\circ} \mathrm{C}$ and $2.5{ }^{\circ} \mathrm{C}$, respectively. This difference potentially influences the comparison. Therefore, the influence of CCM's $T_{g}$ to the WLF parameters ought to be further researched, in order to improve the accuracy of the predictions and to understand the crystallization behaviour of CCM in the deeply supercooled state.

\section{CONCLUSIONS}

This study shows that the storage efficiency of coldcrystallizing material (CCM) can be modelled using WLF equation for long-term heat storage purposes. Because of the unique operational principle of the $\mathrm{CCM}$ heat storage, predicting the storage efficiency is not straightforward. CCM investigated in this work consists of erythritol in sodium polyacrylate matrix, which enables stable supercooling of the material. When the melted CCM is cooled close to the glass transition temperature, the latent heat of erythritol can be stored in the deeply supercooled state for long-term heat storage purposes, for example at $0-10{ }^{\circ} \mathrm{C}$. However, slow crystallization during the storage can decrease the stored latent 
heat content. The discharge is initiated by re-heating the material to cold-crystallization temperature.

In order to predict the storage efficiency of CCM, we measured the overall crystallization rate of two CCM samples under isothermal conditions using differential scanning calorimetry (DSC). The crystallization temperature ranged from $25{ }^{\circ} \mathrm{C}$ to $45{ }^{\circ} \mathrm{C}$. The Avrami equation corresponded the experimental data in a precise and a repeatable manner for a relative crystallinity range from 0.05 to 0.80 . This enabled the exploitation of the Arrhenius and the WLF equation to model the temperature dependence of the crystallization rate constant. Furthermore, it enabled the use of the Avrami equation for the predicted rate constant values. The results showed that the Arrhenius equation significantly overestimated the crystallization rate when compared to the long-term measurements reported in our previous study. On the other hand, the WLF predictions made for CCM-1 corresponded the long-term measurements, indicating validity of the WLF equation. CCM-2's WLF predictions underestimated the storage efficiency, most likely because it displayed larger deviations in the DSC measurements than CCM-1. In order to improve the accuracy of the storage efficiency predictions, larger temperature range ought to be included in the data gathering and the influence of the CCM's glass transition temperature to the WLF parameters ought to be further researched.

The WLF equation appears to yield useful predictions of CCM's storage efficiency, when measurement accuracy is ensured. These predictions can be applied in the selection of feasible CCM composition for further development of the material. For applications, the predictions can be used to estimate the optimal storage period and temperature of CCM. Indeed, if the needed storage time is known, the storage temperature can be adjusted accordingly. This aids the implementation of CCM, as the operational behaviour of a CCM storage unit becomes more predictable and technoeconomic assessments of a year-round long-term heat storage for household heating purposes can be conducted.

\section{ACKNOWLEDGMENT}

This research was funded by the Maj and Tor Nessling Foundation and the HeatStock project funded by Business Finland.

\section{REFERENCES}

11 C. R. Matos, J. F. Carneiro, and P. P. Silva, "Overview of large-scale underground energy storage technologies for integration of renewable energies and criteria for reservoir identification," J. Energy Storage, vol. 21, pp. 241-258, 2019.

[2] S. Koohi-Fayegh and M. A. Rosen, "A review of energy storage types, applications and recent developments," J. Energy Storage, vol. 27, p. 101047, 2020.

[3] A. Safari, R. Saidur, F. A. Sulaiman, Y. Xu, and J. Dong, "A review on supercooling of phase change materials in thermal energy storage systems," Renew. Sustain. Energy Rev., vol. 70, pp. 905-919, 2017.

[4] Y. Zhao, X. Zhang, X. Xu, and S. Zhang, "Research progress in nucleation and supercooling induced by phase change materials," $J$. Energy Storage, vol. 27, p. 101156, 2020.

[5] B. Sandnes, "The physics and the chemistry of the heat pad," Am. J. Phys., vol. 76, no. 6, pp. 546-550, 2008.

[6] G. Englmair, S. Furbo, M. Dannemand, and J. Fan, "Experimental investigation of a tank-in-tank heat storage unit utilizing stable supercooling of sodium acetate trihydrate," Appl. Therm. Eng., vol. 167, no. March 2019, p. 114709, 2020.
7] G. Englmair, C. Moser, S. Furbo, M. Dannemand, and J. Fan, "Design and functionality of a segmented heat-storage prototype utilizing stable supercooling of sodium acetate trihydrate in a solar heating system," Appl. Energy, vol. 221, pp. 522-534, 2018.

[8] S. Puupponen and A. Seppälä, "Cold-crystallization of polyelectrolyte absorbed polyol for long-term thermal energy storage," Sol. Energy Mater. Sol. Cells, vol. 180, pp. 59-66, 2018.

[9] S. Puupponen, V. Mikkola, T. Ala-Nissila, and A. Seppälä, "Novel microstructured polyol-polystyrene composites for seasonal heat storage," Appl. Energy, vol. 172, pp. 96-106, 2016.

[10] K. Nakano, Y. Masuda, and H. Daiguji, "Crystallization and Melting Behavior of Erythritol In and Around Two-Dimensional Hexagonal Mesoporous Silica," 2015

[11] A. Seppälä, A. Meriläinen, L. Wikström, and P. Kauranen, "The effect of additives on the speed of the crystallization front of xylitol with various degrees of supercooling," Exp. Therm. Fluid Sci., vol. 34, no. 5, pp. 523-527, 2010.

[12] B. Sandnes and J. Rekstad, "Supercooling salt hydrates: Stored enthalpy as a function of temperature," Sol. Energy, vol. 80, no. 5, pp. 616-625, 2006.

[13] S. Hirano and T. S. Saitoh, "Long-term performance of latent heat thermal energy storage using supercooling," Proc. ISES World Congr. 2007, vol. I-V, pp. 2741-2745, 2008.

[14] P. F. Barrett and B. R. Best, "Thermal energy storage in supercooled salt mixtures," Mater. Chem. Phys., vol. 12, no. 6, pp. 529-536, 1985.

[15] S. Peng, Y. Hu, J. Huang, and M. Song, "Surface free energy analysis for stable supercooling of sodium thiosulfate pentahydrate with microcosmic-visualized methods," Sol. Energy Mater. Sol. Cells, vol. 208, p. 110390, 2020.

[16] K. Iwase, Y. Nagano, I. Yoshikawa, H. Houjou, Y. Yamamura, and K. Saito, "Cold crystallization in schiff-base nickel(II) complexes derived from three toluidine isomers," J. Phys. Chem. C, vol. 118, no. 48, pp. 27664-27671, 2014

[17] K. Turunen, M. R. Yazdani, S. Puupponen, A. Santasalo-Aarnio, and A. Seppälä, "Cold-crystallizing erythritol-polyelectrolyte: scaling up reliable long-term heat storage material," Appl. Energy, vol. 266, no. 114890, 2020.

[18] C. Schick, R. Androsch, and J. W. P. Schmelzer, "Homogeneous crystal nucleation in polymers," J. Phys. Condens. Matter, vol. 29, no. 453002, 2017

[19] M. Avrami, "Kinetics of phase change. I general theory," J. Chem. Phys., vol. 7, pp. 1103-1112, 1939.

[20] M. Avrami, "Kinetics of phase change. II transformation-time relations for random distribution of nuclei," J. Chem. Phys., vol. 8, pp. 212-224, 1940 .

[21] A. T. Lorenzo, M. L. Arnal, J. Albuerne, and A. J. Müller, "DSC isothermal polymer crystallization kinetics measurements and the use of the Avrami equation to fit the data: Guidelines to avoid common problems," Polym. Test., vol. 26, pp. 222-231, 2007.

[22] E. Piorkowska, A. Galeski, and J. Haudin, "Critical assessment of overall crystallization kinetics theories and predictions," Prog. Polym. Sci., vol. 31, pp. 549-575, 2006.

[23] L. A. Pérez-Maqueda, J. M. Criado, and J. Málek, "Combined kinetic analysis for crystallization kinetics of non-crystalline solids," J. Non. Cryst. Solids, vol. 320, pp. 84-91, 2003.

[24] J. W. Mullin, Crystallization 4th ed. Oxford: Butterworth-Heinemann, 2001 .

[25] P. G. Debenedetti, Metastable liquids - concepts and principles. New Jersey, USA: Princeton University Press, 1996.

[26] J. Mark et al., Physical properties of polymers, 3rd editio. Cambridge, United Kingdom: Cambridge University Press, 2004.

[27] F. Fan and Y. H. Roos, "Glass transition-associated structural relaxations and dpplications of relaxation times in amorphous food solids: a review," Food Eng. Rev., vol. 9, pp. 257-270, 2017.

[28] M. L. Williams, R. F. Landel, and J. D. Ferry, "The temperature dependence of relaxation mechanisms in amorphous polymers and other glass-forming liquids," J. Am. Chem. Soc., vol. 77, no. 14, pp. 3701-3707, 1955

[29] J. D. Ferry, Viscoelastic properties of polymers. New York, USA: John Wiley \& Sons, 1961. 